\title{
Influence and reliability of lower-limb arterial occlusion pressure at different body positions
}

\author{
Luke Hughes $^{1}$, Owen Jeffries ${ }^{1}$, Mark Waldron ${ }^{1}$, Ben Rosenblatt ${ }^{2}$, Conor Gissane ${ }^{1}$, Bruce Paton ${ }^{3}$, Stephen \\ D Patterson ${ }^{\text {Corresp. } 1}$ \\ ${ }^{1}$ School of Sport Health \& Applied Science, St Mary's University, Twickenham, United Kingdom \\ 2 St. George's Park, The Football Association, Burton-Upon-Trent, United Kingdom \\ 3 Institute of Sport, Exercise and Health, University College London, University of London, London, United Kingdom \\ Corresponding Author: Stephen D Patterson \\ Email address: stephen.patterson@stmarys.ac.uk
}

Background: Total arterial occlusive pressure (AOP) is used to prescribe pressures for surgery, blood flow restriction (BFRE) and ischemic preconditioning (IPC). AOP is often measured in a supine position; however, the influence of body position on AOP measurement is unknown and may influence of level of occlusion in different positions during BFR and IPC. The aim of this study was therefore to investigate the influence of body position on AOP. Methods: Fifty healthy individuals (age $=29 \pm 6$ y) underwent AOP measurements on the dominant lower-limb in supine, seated and standing positions in a randomised order. AOP was measured automatically using the Delfi Personalised Tourniquet System device, with each measurement separated by 5 min of rest. Results: AOP was significantly lower in the supine position compared to the seated position (187.00 \pm 32.5 vs $204.00 \pm 28.5 \mathrm{mmHg}, \mathrm{p}<0.001)$ and standing position $(187.00 \pm 32.5$ vs. $241.50 \pm 49.3 \mathrm{mmHg}, \mathrm{p}<0.001)$. AOP was significantly higher in the standing position compared to the seated position $(241.50 \pm 49.3$ vs. $204.00 \pm 28.5 \mathrm{mmHg}, p<0.001)$. Discussion: AOP measurement is body position dependent, thus for accurate prescription of occlusion pressure during surgery, BFR and IPC, AOP should be measured in the position intended for subsequent application of occlusion. 


\section{Influence and reliability of lower-limb arterial occlusion pressure at different body positions.}

Luke Hughes $^{1}$, Owen Jeffries ${ }^{1}$, Mark Waldron ${ }^{1}$, Benjamin Rosenblatt ${ }^{2}$, Conor Gissane ${ }^{1}$, Bruce Paton $^{3}$, Stephen David Patterson ${ }^{1}$

${ }^{1}$ School of Sport, Health and Applied Science, St Mary's University, London, UK, TW1 4SX*

${ }^{2}$ The Football Association. St. George's Park, Burton-Upon-Trent, UK

${ }^{3}$ Institute of Sport, Exercise and Health, 170 Tottenham Court Road, London, UK

Running title: Influence of body position on arterial occlusive pressure

Corresponding author: Dr Stephen Patterson

stephen.patterson@stmarys.ac.uk

* = full postal address

Word count: 3389 (Limit is 3500)

Abstract word count: 206

Number of tables and figures: 2 (Limit is 5) 
1 Abstract

2

3 Background: Total arterial occlusive pressure (AOP) is used to prescribe pressures for surgery,

4 blood flow restriction (BFRE) and ischemic preconditioning (IPC). AOP is often measured in a

5 supine position; however, the influence of body position on AOP measurement is unknown and

6 may influence of the level of occlusion in different positions during BFR and IPC. The aim of

7 this study was therefore to investigate the influence of body position on AOP. Methods: Fifty

8 healthy individuals (age $=29 \pm 6$ y) underwent AOP measurements on the dominant lower-limb

9 in supine, seated and standing positions in a randomised order. AOP was measured automatically

10 using an automated pneumatic tourniquet system, with each measurement separated by 5 minutes

11 of rest. Results: AOP was significantly lower in the supine position compared to the seated

12 position $(187.00 \pm 32.5$ vs $204.00 \pm 28.5 \mathrm{mmHg}, \mathrm{p}<0.001)$ and standing position $(187.00 \pm 32.5$

13 vs. $241.50 \pm 49.3 \mathrm{mmHg}, \mathrm{p}<0.001)$. AOP was significantly higher in the standing position

14 compared to the seated position $(241.50 \pm 49.3$ vs. $204.00 \pm 28.5 \mathrm{mmHg}, \mathrm{p}<0.001)$. Discussion:

15 AOP measurement is body position dependent, thus for accurate prescription of occlusion pressure during surgery, BFR and IPC, AOP should be measured in the position intended for subsequent application of occlusion. 
27

28

\section{Introduction}

The technique of occluding limb blood flow using pneumatic tourniquet cuffs is applied in various settings, such as during surgery (Bussani \& McEwen, 1988), blood flow restriction exercise (BFRE) (Hughes et al., 2017) and ischemic preconditioning (IPC) (Griffin et al., 2017). The level of occlusion achieved by an applied pressure is considered to be an important factor for effective creation of a bloodless surgical field (Bussani \& McEwen, 1988), driving physiological adaptations and preventing full occlusion of arterial blood flow during BFRE (Fahs et al., 2012; Lixandrão et al., 2015), and effectiveness of the IPC stimulus (Cunniffe et al., 2016). The required pressure to reach a desired level of occlusion is influenced by several factors, such as cuff width, limb circumference and blood pressure (Loenneke et al., 2015; Jessee et al., 2016), which makes standardisation of occlusion level difficult using arbitrary pressures. The use of arbitrary pressures in BFRE may influence the amount of fatigue observed during exercise and thus potential adaptations. Furthermore during IPC a standard pressure of $200-220 \mathrm{mmHg}$ is widely used prior to exercise, irrespective of upper- or lower- limb application, which may influence the potential benefit of this technique due to different limb circumference and blood pressure within individuals (Bailey et al., 2012; Barbosa et al., 2014; Patterson et al., 2015). Calculation of arterial occlusive pressure (AOP) involves determination of the pressure required to fully occlude arterial flow to the involved limb (AORN Recommended Practices Committee., 2007). This is most often achieved using doppler ultrasound (Bezerra de Morais et al., 2017) and can be used to prescribe pressure at a relative percentage of AOP to standardise the level of occlusion across cohorts (Laurentino et al., 2012; Hughes et al., 2017; Patterson et al., 2017) . 
49 Occlusion of blood flow is typically applied in one of three positions: supine, seated/semi50 recumbent or standing (Loenneke et al., 2012). However, within the literature it is evident that

51 only a small number of studies measure AOP in the same position that the occlusion stimulus is

52 subsequently applied (Staunton et al., 2015); this may not account for postural influences on

53 hydrostatic pressure (Wilkins, Halperin, \& Litter, 1950; Eiken, 1988). For example, movement

54 of the lower-limb into a dependent position causes changes in hydrostatic pressure, deformation

55 of the vascular bed and an increase in blood flow and pressure within the limb (Trinity et al., 56 2010). Systolic blood pressure has been identified as a major predictive variable of AOP in the 57 upper limbs (Loenneke et al., 2015; Jessee et al., 2016), whereas thigh circumference is the

58 largest predictor in the lower body (Loenneke et al., 2015), thus it is conceivable that posture59 induced changes in blood flow and pressure may affect the pressure required for absolute occlusion of blood flow in that limb.

61

To date, only one study has investigated the influence of body position on AOP measurement (Sieljacks et al. 2018), however this was only in the supine and seated position. Furthermore the reliability of AOP in each of these body positions is unknown. This is problematic as it may result in under/over-estimation of the required pressure, which may have implications for effectiveness in application of an occlusion stimulus. Additionally, heterogeneous changes in AOP between individuals may lead to variation in occlusion stimulus even when prescribed relative to AOP. Thus, the aim of this study was to investigate the influence of different postural positions on AOP measurement. A further aim was to examine the test-retest reliability of AOP at different body positions. 


\section{Materials \& Methods}

73

74

75

76

77

78

79

80

81

82

83

84

85

86

87

88

89

90

91

92

93

94

95

96

97

Participants

Fifty participants (37 males and 13 females) volunteered to participate in the study. Overall mean $\pm \mathrm{SD}$ for age, mass and stature were $29 \pm 6 \mathrm{y}, 77.3 \pm 14.2 \mathrm{~kg}$ and $175.9 \pm 8.4 \mathrm{~cm}$, respectively. All were healthy, active non-smokers free from cardiovascular (CV), pulmonary and metabolic diseases and musculoskeletal injuries in the past 12 months. Participants were asked to refrain from strenuous exercise, caffeine and alcohol in the 24 hours prior to each testing session. All participants provided signed informed consent in compliance with the Declaration of Helsinki, 7th version, October 2013 (World Medical Association, 2013). All protocols were approved by St Mary’s University ethical committee (SMEC_2016-17_121).

\section{Experimental design and procedure}

To examine the influence of body position on AOP, participants attended the laboratory on one occasion. Upon arrival, participant's mass and stature were recorded to the nearest $0.1 \mathrm{~kg}$ and 0.1 $\mathrm{cm}$, respectively. Participants rested for 5 minutes in the supine position on a portable treatment bed, then blood pressure was measured at the brachial artery (Omron M5, Omron Healthcare, Europe B.V., the Netherlands, 14 x $48 \mathrm{~cm})$. Thigh circumference $(\mathrm{cm})$ of the dominant lowerlimb was measured at the midpoint of the distance between the greater trochanter and the lateral condyle of the femur in accordance with International Society for the Advancement of Kinanthropometry (ISAK) guidelines using a flexible steel tape (Lufkin W606PM). Additionally, skinfold thickness (ST) was measured $(\mathrm{mm})$ at this point using skinfold calipers (Harpenden skinfold calipers, British Indicators Ltd, UK). For the experimental procedure, participants 
98

underwent AOP measurements in the dominant leg in a supine, seated and standing position in a within-subjects randomised design. The randomisation was carried out by assigning each participant a number and using publicly available software to allocate the order of conditions (http://www.randomization.com/). Prior to each measurement, participants rested in the required position for 5 minutes to ensure restoration of homeostasis after any movement. For the supine position, participants lay on a portable treatment bed with their arms relaxed by their sides. For the seated position, participants sat upright with their legs straight and the hip flexed at a $90^{\circ}$ angle, assessed using a goniometer. For the standing position, participants stood in the standard anatomical position. Prior to each measurement, participants rested in the required position for 5 minutes to ensure restoration of normal blood flow after any movement (Jessee et al., 2016).

AOP measurement

Restriction of blood flow in the lower-limb was achieved using the Delfi Easy-fit variable contour tourniquet cuff $(11.5 \mathrm{~cm}$ x $86 \mathrm{~cm}$ x $5 \mathrm{~mm})$, connected to a pneumatic cuff inflator (Delfi PTS, Delfi Medical, Vancouver, BC, Canada). The pneumatic tourniquet was equipped with the capability of automatically measuring AOP and calculating the personalised tourniquet pressure, comprised of a dual-purpose personalised tourniquet cuff and a personalised tourniquet instrument containing AOP calculation sensors and software. The pneumatic system connected to the tourniquet cuff increased the cuff pressure in stepwise increments, analysing the pneumatic pressure pulsations in the cuff bladder by the arterial pressure pulsations at each cuff pressure increment, and used these characteristics to determine AOP (McEwen et al., 2015). AOP measurement using this cuff was found to not be clinically or statistically different from using the gold standard doppler technique $(+0.08 \mathrm{mmHg}$ [95\% $\mathrm{CI}-2.66$ to 2.82$]$ for lower limbs $)$ across 
122257 pairs of AOP measurements taken from upper and lower limbs in 143 pre- and post-surgical

123 patients aged 17-86 (McEwen et al., 2015; Masri et al., 2016). This technique of measuring AOP

124 was found to have clinically acceptable accuracy compared to the distal-sensor-based method of

125 automatic AOP measurement, which measures AOP using a sensor located on the most distal

126 phalange of the involved limb (McEwen, Inkpen, \& Younger, 2002). The variable-contour cuff

127 was placed on the most proximal portion of the participant's dominant lower limb directly onto

128 the skin and connected to the pneumatic tourniquet with airtight hose tubing. After 5 minutes of

129 rest, the device was turned on to calculate AOP in the manner described above. The AOP

130 displayed on the pneumatic tourniquet device was recorded for each of the three positions.

131

132

133

134

135

136

137

138

139

140

141

142

143

144

145

146

147

148

Test-retest reliability

To assess the reliability of the pneumatic tourniquet, 10 subjects visited the laboratory at the same time of day, on a second occasion, one week later during which the experimental procedure for the AOP measurement was repeated with the order of positions tested in the same order as they had previously been tested.

\section{Statistical analysis}

Due to non-normal distribution of the supine body position data $(p<0.05)$ which persisted after $\log$ transformations, a non-parametric Friedman test was used to determine if there were differences in AOP across the three different body positions. For any significant differences, Wilcoxon signed-rank pairwise comparisons were performed with Bonferroni correction. Within-subject coefficient of variation (COV) was calculated, and an intraclass correlation coefficient (ICC) test with a two-way mixed effects model was used to determine absolute 
149 agreement to examine the reliability and reproducibility of AOP measurements with the 150 pneumatic tourniquet system. The COV was calculated from the ratio of the standard deviation 151 (SD) and the mean of the two AOP measurements $(\mathrm{CV}=((\mathrm{SD} / \mathrm{mean}) \mathrm{x} 100))$, followed by 152 calculation of the mean (Bezerra de Morais et al., 2017).

153

154

155

156

157

158 159

160

161

162

163

164

165

166

167

168

169

170

171

172

173

174

175

176

\section{Results}

Participants

All 50 participants completed the study with no adverse events. Participants' blood pressure, resting heart rate, thigh circumference and ST are presented in Table 1.

$$
\text { ***Insert Table } 1 \text { here*** }
$$

\section{AOP}

Data are presented as mean $\pm \mathrm{SD}$. AOP was statistically significantly different in the different body positions, $\mathrm{x}^{2}(2)=90.04, \mathrm{p}<0.001$. Post-hoc analysis revealed that AOP in the supine position was significantly lower compared to the seated position $(187.00 \pm 32.5$ vs $204.00 \pm 28.5$ $\mathrm{mmHg}$, respectively, $\mathrm{p}<0.001)$, and the standing position $(187.00 \pm 32.5$ vs. $241.50 \pm 49.3$ $\mathrm{mmHg}$, respectively, $\mathrm{p}<0.001)$. Additionally, AOP in the seated position was significantly lower than in the standing position $(204.00 \pm 28.5 \mathrm{vs} 241.50 \pm 49.3 \mathrm{mmHg}$, respectively, $\mathrm{p}<0.001)$.

Test-retest reliability

For the supine position, the ICC for assessing reliability of the device across two repeated measurements was 0.982 (95\% CI: 0.932 to 0.995$)$ with a COV of $2.94 \%$ (95\% CI: 1.90 to 
$1773.98 \%$ ). For the seated position, the ICC for assessing reliability of the device across two

178 repeated measurements was 0.975 (95\% CI: 0.932 to 0.994$)$ with a COV of $1.82 \%$ (95\% CI: 0.95

179 to 2.69). For the standing position, the ICC for assessing reliability of the device across two

180 repeated measurements was 0.953 (95\% CI: 0.822 to 0.988$)$ with a COV of $2.97 \%$ (95\% CI: 0.89

181 to 5.05$)$.

182

183

184

185

186

187

188

189

190

191

192

193

194

195

196

197

198

199

\section{Discussion}

This study investigated the influence of body position on AOP measurement. The main findings were that lower-limb arterial AOP is body-position dependent. For absolute occlusion of lower limb arterial blood flow, it appears that higher pressures are required in a seated compared to supine body position, and higher pressures are required in a standing compared to seated and supine body position.

The pressure required to fully restrict arterial blood flow to the lower-limb increased from 187 $\mathrm{mmHg}$ to $204 \mathrm{mmHg}$ to $241 \mathrm{mmHg}$ in the supine, seated and standing positions, respectively. This reflects literature demonstrating increases in peripheral blood flow to the extremities (Goetz, 1950) and changes in hydrostatic pressure with different body positions (Wilkins et al., 1950; Eiken, 1988). Elevation of the heart above the limbs when comparing an seated to supine position results in an increase in peripheral blood flow and pooling of blood in the lower limbs due to gravitational forces (Olufsen et al., 2005). Increases in lower-limb local hydrostatic pressure (Wilkins et al., 1950), mechanical deformation of the vascular bed and stimulation of 
200 group III afferent fibres (Trinity et al., 2011) triggers peripheral vasodilation, causing a rise in

201 peripheral blood flow. As there is greater elevation of the heart again in a standing position and a

202 larger effect of gravity, these changes in peripheral blood flow and pressure may be amplified

203 further in a standing position (Olufsen et al., 2005). Studies examining factors influencing AOP

204 in the upper-limb support this, with systolic blood pressure identified as one of the major

205 predictive variables of AOP in the upper-limb (Loenneke et al., 2014; Jessee et al., 2016).

206

207 Implications for BFRE

208

209

These observations suggest that measurement of AOP and application of the occlusive stimulus

210 in different positions would result in undesirable levels of occlusion, which has important

211 implications for application. For example, if AOP is measured whilst standing but occlusion is

212 applied in a seated or supine position, the individual may be exposed to higher levels of

213 occlusion than necessary. Within BFRE, higher pressures have been shown to cause greater CV

214 responses to exercise (Rossow et al., 2012) and may result in full restriction of arterial inflow to

215 the working muscle. It has been speculated that this may increase the risk of ischemic

216 reperfusion injury, peripheral nerve injury or concerning hemodynamic alterations (Kacin et al.,

217 2015; Loenneke et al., 2011; Jessee et al., 2016), particularly when BFRE is used in patients with

218 blood-related conditions such as hypertension and heart disease (Madarame et al., 2010; Cezar et

219 al., 2016). Additionally, although the focus of this study was not on the physiological responses

220 to BFR, higher pressures are known to increase discomfort responses to BFRE (Jessee et al.,

221 2017; Mattocks et al., 2017) and thus could impact upon the clinical utility of BFRE training

222 and patient adherence to clinical rehabilitation programmes. Therefore, accurate calculation of 
223 AOP for pressure prescription is required for selection of the minimum occlusion pressure

224 required to elicit a positive change. It is of note that optimal occlusion pressure is not fully

225 understood, and may vary in different contexts. However, current literature suggests that light-

226 load BFRE $(<30 \%$ 1RM) training protocols benefit from higher occlusion pressures $(80 \%$ vs

227 40\%) (Lixandrão et al., 2015), which would support the importance of accurate AOP

228 measurement for prescription of relative pressures. In contrast when loads are $\geq 30 \% 1 \mathrm{RM}$ there

229 does not appear to be a need to exercise at higher percentages of AOP (Counts et al., 2016).

231 On the contrary, measurement of AOP in a supine position and subsequent application of BFRE

232 in a seated or standing position may result in a lower level of occlusion than desired, or a lack of

233 venous occlusion altogether in situations where low pressures are used (Kubota et al., 2011).

234 Furthermore higher pressures during BFRE result in greater accumulation of metabolic

235 byproducts (Yasuda et al., 2010) hypothesised to be one of the major driving forces of 236 hypertrophic adaptations to light load BFR training (Pearson \& Hussain, 2015; Hughes et al., 237 2017). Insufficient levels of restriction due to inaccurate pressure prescription confounded by 238 body position may reduce the metabolic stress stimulus, which may dampen the hypertrophic

239 BFR stimulus and partially explain reports of ineffectiveness of BFRE. Furthermore AOP may 240 be influenced by time of day, with increased pressure observed as the day progresses, likely 241 brought about by oscillatory changes in changes in blood flow and pressure ((Ingram et al., 242 2017). Therefore measurement position and time of day should be considered by those using 243 BFRE over repeated applications.

245 Implications for IPC 
247 When performing IPC before exercise, the lower or upper limb is occluded at a set arbitrary

248 pressure between 200 and 220mmHg (Bailey et al., 2012; Barbosa et al., 2014; Patterson et al., 249 2015). Furthermore when applying this pressure, participants are either supine (Patterson et al 250 2015) or in a seated position (Marocolo et al., 2017) which may influence the amount of 251 occlusion observed. In the current study the average pressure observed in the supine position was $252<200 \mathrm{mmHg}$, however in the seated position this increased to $204 \mathrm{mmHg}$. This suggests that the 253 normal arbitrary pressures of $220 \mathrm{mmHg}$ should be sufficient to fully restrict blood flow prior to 254 this intervention. However in some studies the pressure used has been $200 \mathrm{mmHg}$ (Barboasa et 255 al., 2014) and as low as $180 \mathrm{mmHg}$ (Cunniffe et al., 2016). In the current investigation 28\% and $25618 \%$ of the participants would not be fully occluded at 200 and $220 \mathrm{mmHg}$ in the supine position, 257 respectively. Furthermore, in the seated position, this number would rise to $60 \%$ and $28 \%$ for 258200 and $220 \mathrm{mmHg}$, respectively. Therefore we recommend the use of AOP to standardise 259 pressures during IPC due to the wide variance in participants and also the wide array of cuffs 260 used to occlude individuals.

Reliability

263 When measuring AOP automatically, it is important that the device used is reliable and 264 consistent across repeated measures to ensure correct prescription of pressure. In this study, the pneumatic tourniquet system appeared to have high reproducibility $(>0.953)$ with a COV of less than $2.97 \%$ across all the body positions examined. These findings are similar to a recent study examining the reliability of doppler ultrasound for calculating total AOP in the upper limbs

268 (Bezerra de Morais et al., 2017). The authors calculated AOP using doppler ultrasound in 13 
269 male volunteers across three repeated measures, reporting an ICC of 0.795 and a COV of $5.6 \%$.

270 Although the present study was in the lower-limbs, we observed greater ICC scores and smaller

271 COVs, suggesting measurement of AOP using the pneumatic tourniquet system may be more

272 reliable than doppler ultrasound. This is may be attributed to the absence of human error;

273 however, this is speculative at present. Nevertheless, other studies have demonstrated similar

274 results. The results of the present study suggest the pneumatic tourniquet system is highly

275 reproducible for measuring lower-limb arterial AOP due to the high ICC values and lower COV

276 scores compared to similar studies in the upper- limb (Bezerra de Morais et al., 2017).

277

278

279

280

281

282

283

284

285

286

287

288

289

290

291

\section{Conclusion}

The findings of the present study have several important clinical implications. Firstly, it appears that AOP is body position-dependent. In BFRE and IPC, AOP must therefore be calculated in the position of exercise to ensure accurate occlusion, while minimising the risk of an adverse CV/neurological event or application of an insufficient BFRE stimulus. Secondly, it appears that the pneumatic tourniquet system can be used to reliably calculate lower-limb AOP. Previously, we highlighted that AOP may change across the duration of a BFRE training study due to various tissue adaptations, such as increases in muscle mass and vasculature adaptations, thus it is important to continually monitor AOP to ensure prescription of the correct pressure (Hughes et al., 2017). Doppler ultrasound tools can be expensive, and repeated measurement of AOP using this technique prior to weekly BFRE training sessions would likely be time-consuming and require considerable skill. This may be exacerbated in a clinical rehabilitation setting such as the 
292 NHS where rehabilitation exercise classes are already time-constrained. However, the pneumatic 293 tourniquet system provides a simple and quick alternative for calculating AOP, and may be 294 implemented on a session-to-session basis. We propose that an actual measurement of AOP is 295 obtained at rest, prior to BFRE, and a percentage of that measurement is used provide a more 296 reliable stimulus (Laurentino et al., 2012; Hughes et al., 2017; Patterson et al., 2017) as this 297 method is still under-utilised by practitioners (Patterson \& Brandner 2017).

In conclusion, the results of this study indicate that for accurate prescription of occlusion 300 pressure in BFRE and IPC applications, body position must be accounted for an AOP measured

301

302 303

304

305

306

307

308

309

310

311

312

313

314

\section{Acknowledgments}

The authors wish to extend thanks to the Delfi Medical team for providing the PTS device for this study.

\section{References}

AORN Recommended Practices Committee. Recommended practices for the use of the pneumatic tourniquet in the perioperative practice setting. 2007 AORN Journal 86:640-655.

Bailey TG, Jones H, Gregson W, Atkinson G, Cable NT, Thijssen DH. Effect of ischemic preconditioning on lactate accumulation and running performance. 2012. Medicine $\&$ Science in Sports \& Exercise 44:2084-9. 
315 Barbosa T, Machado AC, Braz ID, Fernandes IA, Vianna LC, Nobrega AC, Silva BM. Remote

316 ischemic preconditioning delays fatigue development during handgrip exercise. 2015. Scandinavian Journal of Medicine \& Science in Sports 25:356-642014;

Bezerra de Morais AT, Santos Cerqueira M, Moreira Sales R, Rocha T, Galvão de Moura Filho A. Upper limbs total occlusion pressure assessment: Doppler ultrasound reproducibility and determination of predictive variables. 2017. Clinical Journal of Physiology \& Functional Imaging 37:437-441.

Bussani CR, McEwen JA. Improved tracking of limb occlision pressure for surgical tourniquets. 1988. IEEE Translational \& Biomededical Engineering 35: 221-229.

Cezar MA, De Sá CA, Corralo V da S, Copatti SL, Santos GAG dos, Grigoletto ME da S. 2016. Effects of exercise training with blood flow restriction on blood pressure in medicated hypertensive patients. Motriz: Revista de Educação Física 22:9-17.

Counts BR, Dankel SJ, Barnett BE, Kim D, Mouser JG, Allen KM, Thiebaud RS, Abe T, Bemben MG, Loenneke JP. 2016. Influence of relative blood flow restriction pressure on muscle activation and muscle adaptation. Muscle \& nerve, 53:438-445.

Cunniffe B, Sharma V, Cardinale M, Yellon D. Characterization of muscle oxygen response to vasvular occlusion: implications for remote ischaemic preconditioning and physical performance. 2017. Clinical Journal of Physiology \& Functional Imaging 37; 785-793.

334 Eiken O. Effects of increased muscle perfusion pressure on responses to dynamic leg exercise in 
man. 1988. European Journal of Applied Physiology Occupational Physiology 57:772-6.

336 Fahs CA, Loenneke JP, Rossow LM, Thiebaud RS, Bemben MG. Methodological considerations

337 for blood flow restricted resistance exercise. 2012. Journal of Trainology 1:14-22.

Goetz RH. Effect of Changes in Posture on Peripheral Circulation, with Special Reference to Skin Temperature Readings and the Plethysmogram. 1950. Circulation 1:56-75.

Griffin PJ, Ferguson RA, Gissane C, Bailey SJ, Patterson SD. Ischemic preconditioning enhances critical power during a 3 minute all-out cycling test. 2017. Journal of Sports Science doi.org/10.1080/02640414.2017.1349923.

Hughes L, Paton B, Rosenblatt B, Gissane C, Patterson SDSD. Blood flow restriction training in clinical musculoskeletal rehabilitation: a systematic review and meta-analysis. 2017. British Journal of Sports Medicine 51:1003-1011.

Ingram JW, Dankel SJ, Buckner SL, Counts BR, Mouser JG, Abe T, Laurentino GC, Loenneke JP. 2017. The influence of time on determining blood flow restriction pressure. Journal of science and medicine in sport, 20:777-780.

Jessee MB, Dankel SJ, Buckner SL, Mouser JG, Mattocks KT, Loenneke JP. The Cardiovascular and Perceptual Response to Very Low Load Blood Flow Restricted Exercise. 2017. International Journal of Sports Medicine 38:597-603.

Jessee MB, Buckner SL, Dankel SJ, Counts BR, Abe T, Loenneke JP. The Influence of Cuff Width, Sex, and Race on Arterial Occlusion: Implications for Blood Flow Restriction Research. 2016. Sport Medicine 46:913-921. 
355 Kacin A, Žargi TG, Rosenblatt B, Biswas A. Safety Considerations With Blood Flow Restricted

356 Resistance Training. 2015. Annales Kinesiologiae 6:3-26.

357 Kubota A, Sakuraba K, Koh S, Ogura Y, Tamura Y. Blood flow restriction by low compressive 358 force prevents disuse muscular weakness. 2011.Journal of Science and Medicine in Sport $359 \quad 14: 95-99$.

Laurentino G, Ugrinowitsch C, Roschel H, Aoki MS, Soares AG, Neves M, Aihara AY, da Rocha Correa Fernandes A, Tricoli V. Strength training with blood flow restriction diminishes myostatin gene expression. 2012. Medicine and Science in Sports and Exercise 44. $406-412$.

Lixandrão ME, Ugrinowitsch C, Laurentino G, Libardi CA, Aihara AY, Cardoso FN, Tricoli V, Roschel H. Effects of exercise intensity and occlusion pressure after 12 weeks of resistance training with blood-flow restriction. 2015. European Journal of Applied Physiology 115:2471-2480.

Loenneke JP, Allen KM, Mouser JG, Thiebaud RS, Kim D, Abe T, Bemben MG (2015) Blood flow restriction in the upper and lower limbs is predicted by limb circumference and systolic blood pressure. 2015. European Journal of Applied Physiology 2:397-405.

Loenneke JP, Allen KM, Mouser JG, Thiebaud RS, Kim D, Abe T, Bemben MG. Blood flow restriction in the upper and lower limbs is predicted by limb circumference and systolic blood pressure. 2014. European Journal of Applied Physiology 115:397-405.

Loenneke JP, Fahs CA, Rossow LM, Sherk VD, Thiebaud RS, Abe T, Bemben DA, Bemben MG. Effects of cuff width on arterial occlusion: Implications for blood flow restricted 
exercise. 2012. European Journal of Applied Physiology 112:2903-2912.

377

378

379

380

381

382

383

384

385

386

387

388

389

390

391

392

393

394

395

Loenneke JP, Wilson JM, Wilson GJ, Pujol TJ, Bemben MG. Potential safety issues with blood flow restriction training. 2011. Scandinavian Journal of Medicine and Science in Sport 21:510-518.

Madarame H, Kurano M, Takano H, Iida H, Sato Y, Ohshima H, Abe T, Ishii N, Morita T, Nakajima T. Effects of low-intensity resistance exercise with blood flow restriction on coagulation system in healthy subjects. 2010 Clinical Physioliology and Functional Imaging 30:210-213.

Marocolo IC, da Mota GR, Londe AM, Patterson SD, Barbosa Neto O, Marocolo M. Acute ischemic preconditioning does not influence high-intensity intermittent exercise performance 2017 PEER J. Nov 30;5:e4118. doi: 10.7717/peerj.4118

Mattocks KT, Jessee MB, Counts BR, Buckner SL, Grant Mouser J, Dankel SJ, Laurentino GC, Loenneke JP. The effects of upper body exercise across different levels of blood flow restriction on arterial occlusion pressure and perceptual responses. 2017. Physiology \& Behavior 171:181-186.

Masri BA, Day B, Younger ASE, Jeyasurya J. Technique for Measuring Limb Occlusion Pressure that Facilitates Personalized Tourniquet Systems: A Randomized Trial. J Med Biol Eng 2016;36:644-650

McEwen JA, Masri BA, Day B, Younger AS. Development of Personalised Tourniquet Systems using a new technique for measuring limb occlusion pressure. 2015.IFMBE Proceedings 51. 
396 McEwen JA, Inkpen KB, Younger A. Thigh tourniquet safety: Limb occlusion pressure

397 measurement and a wide contoured cuff allow lower cuff pressure. 2002. Surgical

398 Technology 34:8-18

399

400

401

402

403

404

405

406

407

408

409

410

411

412

413

414

415
Olufsen MS, Ottesen JT, Tran HT, Ellwein LM, Lipsitz LA, Novak V. Blood pressure and blood flow variation during postural change from sitting to standing: model development and validation. 2005. Journal of Applied Physiology 99:1523-37.

Patterson SD, Brandner C. The role of blood flow restriction training for applied practitioners: A questionnaire based survey. 2017. Journal of Sports Science 36: 123-130.

Patterson SD, Hughes L, Head P, Warmington S, Brandner C, Blood flow restriction training: A novel approach to augment clinical rehabilitation: how to do it. 2017. British Journal of Sports Medicine 51;1648-1649.

Patterson SD, Bezodis NE, Glaister M, Pattison JR. The effect of ischemic preconditioning on repeated sprint cycling performance. 2015. Medicine and Science in Sports and Exercise $47: 1652-8$

Pearson SJ, Hussain SR. A Review on the Mechanisms of Blood-Flow Restriction Resistance Training-Induced Muscle Hypertrophy. 2015. Sport Medince 45:187-200.

Rossow LM, Fahs CA, Loenneke JP, Thiebaud RS, Sherk VD, Abe T, Bemben MG. Cardiovascular and perceptual responses to blood-flow-restricted resistance exercise with differing restrictive cuffs. 2012. Clinical Journal of Physiology and Functional Imgaing $32: 331-337$. 
416 Sieljacks S, Knudsen L, Wernbom M, Vissing K. Body position influence arterial occlusion

417 pressure: implications for the standardization of pressure during blood flow restricted

418

419

420

421

422

423

424

425

426

427

428

429

430

431

432

433

434

435

436

Staunton CA, May AK, Brandner CR, Warmington SA. Haemodynamics of aerobic and resistance blood flow restriction exercise in young and older adults. 2015. European Journal of Applied Physiology 115:2293-2302.

Trinity JD, McDaniel J, Venturelli M, Fjeldstad AS, Ives SJ, Witman MAH, Barrett-O’Keefe Z, Amann M, Wray DW, Richardson RS. Impact of body position on central and peripheral hemodynamic contributions to movement-induced hyperemia: implications for rehabilitative medicine. 2011. AJP Heart \& Circulatory Physiology 300:H1885-H1891.

Trinity JD, Amann M, McDaniel J, Fjeldstad AS, Barrett-O’Keefe Z, Runnels S, Morgan DE, Wray DW, Richardson RS. Limb movement-induced hyperemia has a central hemodynamic component: evidence from a neural blockade study. 2010. AJP Heart \& Circulatory Physiology 299:H1693-H1700.

Wilkins RW, Halperin MH, Litter J. The Effect of the Dependent Position upon Blood Flow in the Limbs. 1950. Circulation 11:373-379.

World Medical Association. World Medical Association Declaration of Helsinki. Ethical principles for medical research involving human subjects.2013. JAMA 310:2191-2194.

Yasuda T, Abe T, Brechue WF, Iida H, Takano H, Meguro K, Kurano M, Fujita S, Nakajima T. 2010. Venous blood gas and metabolite response to low-intensity muscle contractions with 
437 external limb compression. Metabolism: clinical and experimental, 59:1510-1519.

438

439

440

441

442

443

444

445

446

447

448

449

450 


\section{Table legends}

452 Table 1 Participant anthropometric characteristics (Mean \pm SD)

453

454

455

456 


\section{Table $\mathbf{1}$ (on next page)}

Participant anthropometric characteristics (Mean \pm SD) 
1 Table 1.

2

\begin{tabular}{lcc}
\hline & Males $(\mathbf{n}=\mathbf{3 7})$ & Females $(\mathbf{n}=\mathbf{1 3})$ \\
\hline Age $(\mathrm{y})$ & $29 \pm 6$ & $29 \pm 7$ \\
Stature $(\mathrm{cm})$ & $179.9 \pm 4.8$ & $174.3 \pm 7.9$ \\
Body mass (kg) & $81.8 \pm 13.3$ & $72.7 \pm 11.3$ \\
Systolic Blood pressure & $126 \pm 9$ & $123 \pm 8$ \\
Diastolic Blood Pressure (DBP; & $72 \pm 8$ & $66 \pm 17$ \\
mmHg) & & \\
Resting heart rate (bpm) & $62 \pm 9$ & $61 \pm 9$ \\
Thigh circumference (cm) & $56 \pm 5$ & $53 \pm 7$ \\
ST (mm) & $14.1 \pm 5.8$ & $17.8 \pm 8.6$
\end{tabular}

3

4 